\title{
Calidad asistencial y cronicidad en los programas de mantenimiento con agonistas opiáceos
}

\author{
Juan José Fernández Miranda \\ Responsable de la Unidad de Tratamiento de Toxicomanías. Servicio de Psiquiatría y Salud Mental. Área III (Avilés). \\ $\mathrm{S}^{\circ}$ de Salud del Principado de Asturias (SESPA). ) \\ Enviar correspondencia a: \\ Juan José Fernández Miranda. Unidad de Tratamiento de Toxicomanías. C/ Llano Ponte 49 bajo. 33400-Avilés. \\ Tel 985510620. Fax 985542992. e-mail: miranvilla@inicia.es
}

\section{RESUMEN}

En salud mental, la "cronicidad" ha pasado de necesidad de largos tiempos de cuidados a déficits de ajuste entre necesidades de los pacientes y oferta sociosanitaria comunitaria. Los mantenimientos con opiáceos han tendido a la reducción de daños, con gran efectividad, pero arrastrando la etiqueta de "cronificadores". Aunque la masificación puede alargar permanencias, los mantenimientos largos son soporte prolongado para romper la "cronicidad" de los problemas de la adicción. A mayor efectividad, menor será la cronicidad, entendida como dependencia del tratamiento, que no propiciaría la autonomía para una vida sin drogas. La calidad pasaría por dar a los pacientes lo que necesitan o demandan, disminuyendo sus discapacidades y cronificación.

La ventaja de un sistema de calidad es que su gestión implica mejora continúa. Y esta pasa por la completa integración de los PMMs en la red sanitaria general, consiguiendo así una atención integral e integrada. Y por la definición de subprogramas, desde la abstinencia a lo paliativo, y de procedimientos en cada uno de ellos, además de diversificar niveles asistenciales. Es necesaria la adaptación a nuevas necesidades en viejos consumidores y a nuevos usuarios, actualizando objetivos e indicadores, identificando variables relacionadas con efectividad y cronicidad. El éxito de los PMMs para contener el sida no debe hacer olvidar que son verdaderos tratamientos biopsicosociales de efectividad contrastada, y que deberán de ser por ello adecuados a este objetivo.

Palabras clave: mantenimiento con metadona, cronicidad, calidad asistencial, efectividad.

\begin{abstract}
In mental health, 'chronicity' has moved from the need for long periods of care to matching patients' needs to community social and health resources. Opiate maintenance treatments have tended to harm reduction, with great effectiveness, but have been dogged by being labelled as provoking chronicity. Although crowding may involve longer treatment duration, lengthy maintenance merely means prolonged support to break the chronicity of drug use problems. Greater effectiveness implies less chronicity, this being understood to mean dependence on treatment, which would not foster personal autonomy for a life free of drugs. The quality of treatment should give patients what they need or ask for, reducing their disabilities and chronicity.

The advantage of a quality system in addiction treatments is that it implies a continuous improvement. And this improvement means the complete incorporation of MMTs in the general health system, thereby achieving an overall and integrated attention. Subprogrammes must be defined, ranging from abstinence to palliative care, with their procedures, and also matching them with different assistance levels. It is also necessary to adapt to new needs by 'old' users and to the new ones, updating goals and outcome indicators, and trying to identify variables related to effectiveness and chronicity. The success of MMTs in containing AIDS should not allow us to forget that they are true biopsychosocial treatments, of proven efficacy and, consequently, should be perfectly suitable for this objective.
\end{abstract}

Key words: methadone maintenance, chronicity, quality of assistance, effectiveness 


\section{CONCEPTO DE CRONICIDAD Y ADICCIONES. LOS PROGRAMAS DE MANTENIMIENTO.}

H istóricamente, el concepto de cronicidad se ha basado en varios criterios. El primero, el diagnóstico, ya que algunos diagnósticos tienen precisamente como criterio la larga duración. También la propia duración, ya porque sea larga o porque pase por recurrencias frecuentes. El nivel de discapacidad, es decir, la afectación para la actividad personal, social o laboral ha sido otro criterio considerado. Conviene recordar que la discapacidad se puede clasificar como: primaria (producto directo de la enfermedad); secundaria (reacciones psíquicas adversas a la enfermedad); y terciaria (respuesta social a la enfermedad). Y también que el diagnóstico es insuficiente para seguir la evolución de una enfermedad de larga duración: hay que conocer las consecuencias de la enfermedad como base de un pronóstico.

En salud mental se ha concebido la cronicidad de diferentes formas, pasando de ser la necesidad de largo tiempo de cuidados a los pacientes, muchas veces residenciales y casi con objetivos paliativos, a considerarla como los déficits de ajuste entre las variadas y complejas necesidades de los pacientes y la oferta del sistema sociosanitario comunitario .

La intervención en drogodependencias no se diferencia de otros ámbitos sanitarios y sociales, aunque precisa realizar mas especialmente un análisis del contexto donde debe inscribirse la atención a los problemas relacionados con las drogas, es decir, las condiciones sociales y políticas que determinan las líneas de actuación. Desde el paradigma de la abstinencia que condicionó los modelos de intervención durante los 80 hasta la redefinición de las políticas de intervención en los 90 , orientadas claramente hacia la reducción de los daños y a la mejora de la calidad de vida de los usuarios de drogas, se han producido múltiples estrategias con desigual eficiencia. Ciertamente, ha sido un proceso que se ha caracterizado por su alto dinamismo (poco más de un cuarto de siglo de historia), fuerte impacto en la calidad de vida de las personas y de la comunidad, y que ha tenido un punto de inflexión tras la eclosión de la infección por el virus de la inmunodeficiencia humana (1).

En concreto, la evolución de los programas de mantenimiento con agonistas opiáceos (PMAOs) en los 90 ha tendido claramente a la reducción de daños, entendida sobre todo como contención y control sociosanitario. En este sentido, pueden valorarse como muy efectivos, a pesar de la masificación que ha supuesto tal política, y que es consecuencia del primero de los objetivos específicos: el contactar con el mayor número posible de adictos a heroína. No obstante, esta expansión de los PMAOs ha supuesto quizás que no se consideren como "verdaderos" tratamientos de la adicción, alejándose de la filosofía rehabilitadora y el diseño globalizador que Dole y Nyswander propusieron originalmente. En definitiva, en los 90 se primó, por criterios de salud pública, la "cantidad" a costa de la "calidad" (2).

Esto supuso en muchos casos precariedad de recursos, especialmente humanos, aunque también materiales (edificios sin las condiciones necesarias para una atención no masificada, viejos o en lugares de ciudades poco accesibles; escasa informatización; etc.). La falta de personal, las diversas dependencias administrativas de los equipos y en ocasiones su precariedad laboral, su variopinta configuración, las dificultades para la formación continuada y el reciclaje profesional, y por último la escasa consideración profesional en general, fueron definitorios de los PMAOs durante su expansión en la década pasada.

Además, la adscripción de los programas con sustitutivos a redes específicas en la mayoría de las comunidades autónomas, y en muchos casos dentro de estas con dotaciones presupuestarias muy por debajo de otros abordajes mucho mas onerosos y sin demostrada superior eficacia (3) supusieron, por un lado, la dificultad de coordinación con la red sanitaria general (atención primaria, salud mental, medicina interna, etc...), y por otro que los programas de mantenimiento con metadona (PMMs) tampoco ofrecieran recursos propios de apoyo psicosocial, inherentes a su diseño original, complementarios a la mera dispensación y soporte farmacológico. En definitiva, no se dio ni lejanamente una equiparación a la atención sanitaria de otras patologías, siendo el máximo exponente de esto la señalada no integración en general en el sistema sanitario normalizado.

Incluso respecto al propio soporte farmacológico es necesario recordar también las dificultades para emplear otros opioides diferentes a la metadona en los PMAOs, que teóricamente la ley permite, fruto fundamentalmente del escaso interés de las administraciones sanitarias por la investigación en este campo durante los 90 (Por ello, hablar de PMAOs equivalió y aún equivale a hablar de PMMs). Y es que, en general, fue muy escasa la evaluación con metodología rigurosa en esos años (2), y lenta la trasferencia de conocimientos derivados de la "medicina basada en la evidencia", además de una casi nula introducción de la "gestión clínica".

Mas grave fue, especialmente a principios de la década, la indefinición de objetivos y metodología de estos tratamientos, a pesar de su rápido desarrollo, y la inexistencia de guías clínicas que orientasen intervenciones comunes y contrastadas científicamente. A esto hubo de añadirse la escasa planificación asistencial, en muchos casos marcada por respuestas muy condicionadas socialmente (1).

Todo esto supuso un etiquetaje negativo de los PMMs, no solo por la población general, sino por par- 
te de numerosos profesionales del ámbito psicosocial (2). Con ello, la estigmatización de los heroinómanos, ya alta en general, se multiplicó en los tratamientos con sustitutivos, siendo palpable el rechazo en vecinos y profesionales relacionados de algún modo con los centros que llevaban adelante PMMs.

Sin embargo, a pesar de lo dicho, y aunque es cierto que la masificación de los PMMs puede condicionar permanencias mas largas en los mismos, conviene recordar que es mucho mas discutible que los mantenimientos prolongados signifiquen "cronicidad" siempre, y no simplemente necesidad de soporte farmacológico prolongado para romper precisamente la "cronicidad" de los múltiples problemas relacionados con la adicción. Estas situaciones son mas claras en las personas en mantenimiento con opioides, en general de largo recorrido en la dependencia y con alta discapacidad.

Probablemente por lo anterior, los PMMs han sido difíciles de evaluar (2), dada la inicial heterogeneidad de sus objetivos y el perfil de pacientes incluidos (alto, medio, bajo umbral). En realidad, ha sido la metadona la que ha definido los "programas", y no los objetivos, como cabría esperar. De todas formas, la diversidad de criterios de indicación de tratamiento, alta terapéutica, etc., han sido comunes para todas las modalidades terapéuticas de la adicción a opioides en la década de los 90, aunque con clara tendencia a homogenizarse y concretarse. Aún así, los PMMs han arrastrado la etiqueta de "cronificación" en la dependencia, a pesar de ser éste un término aún debatido en salud mental en general y en adicciones en particular, y de que las múltiples evaluaciones realizadas los muestran como altamente efectivos, incluso por encima del resto de tipos de tratamiento (3).

Actualmente, el reto de los PMMs, y de las intervenciones en drogodependencias en general, es concretar las variables relacionadas con su efectividad (tanto las relativas al usuario como al propio tratamiento) y conocer los factores de cronicidad en ellos, entendidos como aquellos que impiden mejorías sustanciales durante los tratamientos que permitirían la completa autonomía personal, determinando así las mejoras en el diseño y atención de PMMs para disminuirla.

\section{EFECTIVIDADY CRONICIDAD EN PMAOS}

La efectividad de los PMAOs está estudiada y contrastada suficientemente, Así, las conclusiones de la Revisión Cochrane 2002 "MMT vs no opioid replacement therapy for opioid dependence", son categóricas: "El mantenimiento con metadona es una intervención efectiva en el tratamiento de la dependencia de heroína al retener a los pacientes en tratamiento y disminuir el uso de heroína mejor que tratamientos que no utilizan la sustitución opioide. No hay diferencias estadísticamente significativas sobre un superior efecto en la [disminución de la] actividad criminal" (3).

Las clásicas variables de efectividad de los tratamientos de las adicciones en general, y en los PMMs en particular son $(2,4,5,6)$ : retención en tratamiento, consumo de drogas, morbimortalidad, empleo, criminalidad y calidad de vida. No obstante, es llamativo que las evaluaciones de PMM se han centrado en medir variables "duras", pero no se han detenido en general a conocer el nivel de calidad de vida obtenido. Aunque el término "calidad de vida" es de difícil precisión, la percepción subjetiva del paciente como complemento a la medida de las variables objetivas ofrece la posibilidad de plantear objetivos consensuados con él, además de una evaluación conjunta de los resultados. También ayuda a identificar déficits en áreas de funcionamiento y, por ende, la severidad de la adicción, facilitando así la identificación de grupos de riesgo y la planificación de medidas preventivas (7).

Pero el gran reto de la evaluación de los PMMs es conocer las variables relacionadas con su efectividad, o su contrario, la "cronicidad", ya sean tanto las relativas al sujeto como las concernientes al propio tratamiento. Las características del sujeto que pudiesen predecir el resultado del tratamiento han sido investigadas sin que existan conclusiones relevantes al respecto salvo la de que son menos importantes que las características de los tratamientos. Magura et al (8) y Kosten et al (9), discrepan sobre la influencia de los antecedentes criminales, al igual que Mclellan et al (10), Kosten et al (9), Maddux et al (11) y Magura et al (8) lo hacen sobre la de la severidad de la adicción. Tampoco Yancovitz et al (12) y Dunteman et al (13) coinciden sobre la del uso previo de cocaína. Tschan y Tueller (14) y Fdez. Miranda et al (15) hallan relación entre deterioro somático y consumo de drogas, pero Grella et al (16) dicrepa del anterior, ya que muestran que los VIH positivos tienen mas probabilidades de abandonar el tratamiento. La alta prevalencia de trastornos psiquiátricos en pacientes adictos es un hecho ampliamente constatado, y para muchos autores empeoran el pronóstico $(9,16,17,18,20)$, aunque Friedman y Glickman (21) muestran lo contrario. No obstante, parece que los problemas psiquiátricos mejoran notablemente al estabilizarse en el PMM $(17,18,20)$.

Aún estando el debate abierto, las variables relativas al sujeto que ofrecen mayor consenso son la existencia de psicopatología asociada y el consumo previo de cocaína, que se relacionan con una peor evolución. No obstante, autores como Maddux et al (22) no encuentran ninguna variable pretratamiento predictiva. Sin embargo, y al contrario que las variables relativas al sujeto, existe un amplio consenso entre los investigadores en señalar que las características del trata- 
miento tienen una importante incidencia en la efectividad del mismo, siendo la dosis la más ampliamente estudiada. En general, los factores que correlacionan mejor con resultados satisfactorios son la dosificación adecuada, el tiempo en tratamiento, la oferta asistencial amplia y la flexibilidad del programa.

Los criterios actuales sobre dosis de metadona abogan por su individualización, situándose en general el intervalo efectivo de mantenimiento entre 60-100 mg. Dosis menores empeoran el pronóstico, como han apuntado numerosos autores $(4,5,23,24,25)$. Sin embargo, Maddux et al (22) apenas encuentran diferencias entre pacientes con dosis superiores o inferiores a $50 \mathrm{mg}$, siendo la permanencia en tratamiento más de tres años la que se relaciona con un marcado descenso de consumos.

La adherencia al tratamiento es una de las características de los PMM. Es necesaria la permanencia en los mismos un tiempo suficiente (en general de más de dos años) para conseguir mejoras relevantes en la vida del adicto $(4,15,27)$. También la amplitud de la oferta asistencial es un factor clave. Los PMM con una orientación terapéutica integral deben dar respuesta global a las necesidades del usuario, y disponer por tanto de servicios propios o circuitos de derivación ágiles, con abordaje de las conductas adictivas, control de las patologías orgánicas y psiquiátricas asociadas y de las carencias sociales $(5,26,27)$. Así, el consumo de heroína no sólo se relaciona con el factor dosis, sino con la calidad en general de los servicios ofertados por el PMM $(8,10)$. Entre los factores que aumentan la retención destacan la accesibilidad al centro y a su personal, horario amplio e interferencia mínima en las actividades cotidianas del paciente, facilitando las tomas en casa $(6,27)$.

Es evidente que, en lo que respecta a la calidad de servicios prestados, a pesar de la inexistencia de estudios sólidos al respecto en España, la mejora de los mismos aumentaría la efectividad de los PMMs en nuestro país. Según vimos, al estar fuera del circuito asistencial normalizado las derivaciones se complicaban; y el estar poco dotados presupuestariamente dentro de las redes específicas de atención a las adicciones, los servicios propios eran escasos, y la integración de pacientes de PMMs en otros recursos para drogodependientes "con objetivo abstinencia" dificultada por el rechazo de los propios profesionales en muchos casos. La mejora de estas prestaciones cabe pensar que aumentaría su efectividad y disminuiría la "cronicidad" ligada a las deficientes respuestas asistenciales dadas a sus múltiples problemas psicosociales, es decir, a las "variables relativas al tratamiento".

\section{CONCEPTO DE CALIDADY SU APLICACIÓN A LAS ADICCIONES}

La calidad puede entenderse como el compromiso de todas las personas de la organización sanitaria en mejorar sus actuaciones aplicando todo el conocimiento disponible y aprovechando las oportunidades de mejora para satisfacer a los usuarios con eficiencia. Se han descrito varias dimensiones de la misma: científico-técnica (competencia profesional); calidad percibida (por el usuario), eficiencia (coste-efectividad), satisfacción de los profesionales y accesibilidad (28).

Es importante recordar que los cambios asistenciales en adicciones han sido muchos y en general poco planificados y poco basados en evidencias científicas. Así, en los 80/90 hubo indefinición de objetivos y metodología, no existiendo guías clínicas. Además, la evaluación con metodología rigurosa fue escasa, y la trasferencia de conocimientos y de "medicina basada en la evidencia" lenta. Esto supuso escasa planificación asistencial, con respuestas a los diferentes problemas muy condicionadas socialmente y con pobre introducción de la gestión clínica. También se mantuvo un etiquetaje negativo de los PMMs. Y, finalmente, no se dio una equiparación de la atención sanitaria a las adicciones con la de otras patologías, p. ej. integrándose completamente, a todos los niveles, en el sistema sanitario normalizado (27).

Por todo lo anterior, las ventajas de un sistema de calidad en adicciones son mas aún que en otros ámbitos sanitarios. Y es que el establecimiento de cualquier sistema de gestión de la calidad en drogodependencias, como en cualquier otra área del sector sociosanitario, implica una filosofía previa de mejora continua. Además, implementar un sistema o programa de gestión de la calidad conlleva un conjunto de elementos estructurales y actividades que tienen como finalidad la mejora de la calidad (28).

Por ello, es necesaria la consolidación de un proceso de mejora continúa de la calidad asistencial de las prestaciones que genera la intervención en drogodependencias. En este sentido, el diseño y establecimiento de un sistema de gestión de la calidad es clave. Dicho sistema, consensuado y llevado a cabo por todos los profesionales, podría ser auditado y certificado por una entidad externa (1). Ello supone un notable avance en la garantía de una adecuada atención a las necesidades y demandas que precisan los pacientes, así como a la propia sociedad.

La metodología para su implantación pasa por el establecimiento de objetivos de la calidad que deben ser conseguidos por toda la organización, de procedimientos de detección de desviaciones con la aplicación de acciones correctoras y preventivas y de auditorias periódicas, así como por la introducción sistemática de formación y divulgación del propio sistema de la cali- 
dad $(1,28)$. Todo esto va a permitir la coordinación entre niveles de atención (accesibilidad), el conocimiento del punto de vista del usuario (satisfacción del cliente), la detección y corrección de errores (calidad científicotécnica) y, en definitiva, mayor eficacia y eficiencia.

Algunos criterios generales para avanzar en el camino hacia la calidad en adicciones pasarían por la organización de los centros, la planificación del sistema y su revisión por la dirección, la consideración de los derechos del paciente y de la ética de la organización, la investigación, la formación del personal, el mantenimiento de los equipos, la elaboración de vías clínicas, la realización y revisión de programas y el diseño y desarrollo de otros nuevos.

Para desarrollar el sistema de calidad, es importante concretar un "manual de la calidad", que no es otra cosa que la descripción de los procedimientos de gestión que recogen los requisitos de la norma e instrucciones operativas. Sus objetivos son el protocolizar todas las actuaciones y programas de intervención y el diseñar e implantar un sistema de aseguramiento de la calidad, (ISO 9002, EFOM, JCHQ, etc.) $(1,28)$. Su implantación conlleva identificar al profesional y al usuario para todas las actividades; interrelacionar todas las áreas funcionales de la organización y realizar un reparto de actividades en procesos; y regular la competencia, autoridad y relaciones entre distintos recursos y entre puestos de trabajo mediante soportes documentales en forma de procedimientos generales y protocolos específicos, consensuados con las personas que los van a desarrollar.

\section{MEJORA DE LA CALIDAD EN LOS PMAOS}

Todo lo referente a la calidad tiene una traducción concreta, aplicable a los PMAOs. En este sentido, y basándose en la clásica distinción de Donabedian entre estructura, proceso y resultados (29), podemos definir algunos cambios en ellos que mejorarían su calidad.

\section{A) A nivel de procesos:}

Es imprescindible la integración completa de la asistencia a los adictos en general, y a los pacientes en PMAOs en particular, en la red sanitaria general, y específicamente la ubicación de su atención especializada en la red de salud mental, entendiendo la dependencia como un trastorno mental y del comportamiento mas, y considerando la elevada prevalencia de trastornos duales. Solo así se conseguirá una atención integral e integrada, que disminuiría las dificultades de derivación y coordinación con otros recursos sanitarios, abordaría globalmente los problemas de los heroinómanos, con continuidad de cuidados, y finalmente reduciría el etiquetaje negativo. $Y$ especialmente te- niendo en cuenta que lo anterior se amplifica en los que se encuentran en programas con sustitutivos.

Además, debe lograrse una definición de subprogramas con agonistas opiáceos, desde la abstinencia a lo paliativo. $Y$ de niveles asistenciales: la metadona no puede definir un programa o tratamiento como lo ha hecho hasta ahora, por lo que pueden desarrollarse programas de mantenimiento con diferentes objetivos en atención primaria, salud mental, salud pública, etc (19). Es necesaria también una concreción de procesos y de procedimientos en cada uno de estos subprogramas, señalando los profesionales necesarios para desarrollarlos y sus funciones, así como incorporarles una evaluación terapéutica sistematizada. Solo así se consolidarán como "verdaderos tratamientos", avalados por la epidemiología y la psicobiología, y con una sólida metodología de intervención derivada de la "medicina basada en la evidencia" científica.

Para lo anterior, la introducción de herramientas de gestión clínica (cartera de servicios, vías clínicas, monitorización de indicadores, etc) se hace necesaria. También la continuidad de cuidados, aprovechando $\mathrm{p}$. ej. el "case managment". Esto serviría, entre otras cosas, para evitar la delegación del resto de niveles asistenciales ante cualquier actuación sociosanitaria en los PMMs, como ocurrió con frecuencia en la pasada década. Y, por último, es inevitable la adaptación de las intervenciones con "viejos consumidores" (p.ej. debidos a la mayor supervivencia $\mathrm{VIH}$, al incremento del uso de cocaína, etc.) y con los "nuevos usuarios" (policonsumidores jóvenes, integrados socialmente y con elevada comorbilidad psiquiátrica).

\section{B) A nivel de resultados:}

Aunque los PMMs han demostrado ser eficaces no solo en disminuir daños asociados al consumo, sino también en lograr el abandono del uso de heroína, convendría actualizar los indicadores de su efectividad, ya que aún son los de los 90, especialmente en cuanto a la comorbilidad somática (en disminución) y psiquiátrica (en aumento). Pero lo importante será la identificación de variables relacionadas con dicha efectividad y con la cronicidad, teniendo en cuenta la importancia de la calidad de vida y de la comorbilidad psiquiátrica como indicadores. Finalmente, es imprescindible el desarrollo de estudios de coste-efectividad, entre tipos de PMAOs, y entre éstos y otros tratamientos de la dependencia de opioides, para mejorar la eficiencia de la atención a los heroinómanos.

Todo lo dicho contempla que a mayor efectividad de los PMAOs menor será la cronicidad en ellos, entendida como dependencia del propio programa al no lograr este dotar a sus usuarios de la necesaria autonomía en sus vidas sin problemas derivados del uso de drogas. Es decir, la calidad pasaría por proporcionar a los pacientes aquello que realmente necesitan 
o demandan, lo que disminuiría sus discapacidades y su cronificación. En resumen, efectividad y cronicidad serían dos caras de la misma moneda. En este sentido, el conocimiento de los factores de efectividad/ cronicidad harían mas fácil el adecuar los PMMs a las necesidades de sus usuarios, y ajustarlos a los diferentes subprogramas ("matching") $(27,28)$.

También es fundamental el propiciar estudios de coste-efectividad en nuestro país, en los que previsiblemente los PMAOs se muestren superiores a otras modalidades terapéuticas en el tratamiento de la dependencia de opioides, y a partir de sus resultados distribuir los recursos generales del tratamiento de los heroinómanos con criterios científicos y no de "imagen social" de los diferentes abordajes. Esto serviría para facilitar la mejora de la calidad en los PMAOs, ya que ésta siempre tiene un coste económico extra si se parte de una situación de precariedad como la de los PMMs hasta ahora.

\section{C) Cambios futuros de los PMAOs}

La asistencia a los adictos debe asemejarse a la de otras ofertas sanitarias, aún cuando actualmente no es uniforme, debido a la diversidad de los equipos terapéuticos y al escaso seguimiento de protocolos y guías clínicas. El abordaje de las adicciones debe equipararse, en filosofía y medios, al del resto de los pacientes con patologías crónicas de los servicios de salud mental, y en ellos debe realizarse fundamentalmente el tratamiento especializado, biopsicosocial, de la "dependencia". El objetivo final es conseguir una atención global e integrada en la red sanitaria general.

Los PMMs deben ser coordinadores del proceso rehabilitador del drogodependiente, pero no asumir todas las intervenciones en exclusiva. Debe evitarse la delegación del resto de los dispositivos sociosanitarios. Por otro lado, aunque debe racionalizarse, el "problema" de los PMMs no tiene que centrarse en la dispensación, sino que deben definirse subprogramas con diseños (objetivos, perfiles de inclusión, metodología de tratamiento., etc.) diferentes, sustentados en la "medicina basada en la evidencia", y evaluables. Para ello, habrá de incorporarse la gestión clínica y por procesos/programas, estableciéndose sistemas de calidad.

Quizás un modelo operativo de intervención especializada en adicciones con calidad sería el de unidades funcionales dentro de los equipos de salud mental, que desarrollarían los programas específicos para el abordaje de las dependencias, entre los que estarían aquellos que utilizasen sustitutivos. Los equipos de estas unidades estarían compuestos por profesionales de la salud mental especializados en adicciones. Esto facilitaría además el abordaje de la cada vez mas preocupante patología dual. En definitiva, de este modo se combinaría la normalización e inespeci- ficidad funcional de la atención con la capacidad y los conocimientos especializados.

Además, es imprescindible la adaptación a nuevas demandas en "viejos consumidores" y a las de los "nuevos usuarios" de heroína. Por esto es necesario el replanteamiento de nuevos objetivos y nuevos indicadores. Ello ayudaría a la identificación de variables relacionadas con efectividad y cronicidad, y el costeefectividad. Conviene recordar que, con relación a la eficiencia, los indicadores disponibles (retención, uso de drogas, SIDA y otras patologías somáticas, mortalidad, delincuencia, etc.) deben revisarse y actualizarse. Por último, los PMAOs, tenderán a converger y homogenizarse en España y en Europa, lo que influirá en la evaluación de su calidad.

Pero sobre todo, el éxito de los PMMs para neutralizar el VIH no debe hacer olvidar que son un recurso muy útil no solo para "reducir daños", sino también para regular estilos de vida y mantener la abstinencia. Por ello, y como verdaderos tratamientos biopsicosociales de la dependencia de opioides, de efectividad contrastada, deberán de ser predominantes en su abordaje, independientemente de la epidemia del SIDA que los expandió.

\section{CONCLUSIONES}

Debido a la escasa estructuración y homogenización metodológica de la intervención en los PMAOs y de sus carencias en cuanto a recursos humanos y materiales durante la década de los 90, a pesar de su enorme expansión, la necesidad de implantar programas de mejora de la calidad se hace muy acuciante. Solo así se conseguirá que estos tratamientos tengan la misma consideración profesional y social que el resto de abordajes sanitarios de otras patologías.

La asistencia a los adictos debe asimilarse a la de otras patologías, en cuanto a recursos asistenciales. El abordaje debe equipararse al del resto de los pacientes con patologías crónicas de salud mental. Y ahí debe realizarse, a nivel especializado, el tratamiento biopsicosocial de la dependencia, entendida como trastorno mental y del comportamiento. Y en este contexto integrar los programas con sustitutivos, concebidos como un soporte farmacológico que permite intervenciones psicosociales de largo alcance, incluidos el mantenimiento de la abstinencia y la total rehabilitación.

Por esto, la primera condición básica para hablar de calidad en los PMAOs, a nivel de proceso, es su plena inclusión en la red sanitaria general. Esto implica y facilita conseguir una atención integral e integrada y así mejorar la asistencia, ya que ayudaría a disminuir los problemas de coordinación entre niveles asistenciales y de estigmatización de los pacientes. En este sentido, 
la tendencia en los últimos años parece decantarse por la integración de las redes específicas en los Servicios de Salud Mental, siguiendo un modelo que algunas CC.AA. implantaron ya desde los años 80 (p. ej. Asturias), en el que la atención a los pacientes en PMMs se hace desde los servicios de salud mental por equipos especializados en adicciones. No obstante, esta tendencia no esta generalizada, con lo que se mantendrán diferencias entre las comunidades autónomas no solo desde el punto de vista de la accesibilidad y equidad sino también desde la composición profesional de los equipos de tratamiento y de los protocolos de intervención, dificultando la consolidación como "verdaderos tratamientos" de los PMAOs.

Los PMMs, en este contexto de integración, podrán ser coordinadores del proceso rehabilitador, pero no asumir todas las intervenciones con los heroinómanos como ha ocurrido en muchos momentos en la década anterior. Su integración completa en la red sanitaria normalizada disminuirá la delegación del resto de los dispositivos sociosanitarios.

La segunda condición fundamental de mejora de los PMMs es que deben concretarse subprogramas con objetivos (paliativos, abstinencia, etc.), perfiles de inclusión y diseños de tratamiento diversos, adscritos a diferentes niveles asistenciales (Atención Primaria de Salud, Servicios de salud Mental, etc.), avalados por la "medicina basada en la evidencia" y evaluables. Habrá de incorporarse la gestión clínica y por procesos/ programas, definiendo por tanto los objetivos de cada subprograma, los procesos, los profesionales implicados y sus funciones, los procedimientos a seguir en cada proceso, y estableciendo sistemas de evaluación, especialmente de resultados. En definitiva, diseñar unidades funcionales de atención a los adictos, dentro de las que se encontrarían los diferentes subprogramas de tratamiento con sustitutivos. Todo ello comporta quizás en muchos casos mayores costes de los que hasta ahora han supuesto los PMAOs, especialmente si se asume su condición originaria de abordaje biopsicosocial, y no solo "bio" (farmacológico), y su objetivo rehabilitador.

Otro importante reto de la gestión en el tratamiento de la adicción opiácea es, una vez constatada sobradamente la efectividad de los PMMs no solo como "disminuidores de daños" y contenedores de la infección por el VIH, confirmar su mayor coste-efectividad respecto a otros abordajes históricamente mas "respetados", pero que no han demostrado mayor eficacia y sí un elevado coste. La distribución de recursos a los tratamientos de la dependencia de opiáceos en función de su coste-efectividad y no de su "imagen social" es la tercera fundamental condición par la mejora de su calidad. Mas que una condición, será el medio por el cual se ajusten recursos a los PMAOs que permitan la mejora de su calidad. Cabe pensar que trasvasar mas recursos a los PMMs, que con pocos ya son claramente mas eficaces en el tratamiento de estos adictos que otras modalidades, lograría la altísima efectividad que demostraron las primeras evaluaciones sobre ellos en los 60, cuando su orientación era claramente rehabilitadora y dotada con recursos apropiados para ese fin.

En definitiva, la mejora de la calidad en los PMAOs disminuirá la cronicidad de los problemas de los pacientes heroinómanos, aunque siempre considerando que el concepto de mantenimiento pasa por el soporte biopsicosocial del proceso rehabilitador por tiempos largos en general.

\section{REFERENCIAS}

1. Mendieta Caviedes S. Nuevas estrategias regionales sobre drogas. Trastornos adictivos 2001; 3: 199-204.

2. Fernández Miranda JJ. Efectividad de los programas de mantenimiento con metadona. Una revisión de los estudios de evaluación de sus resultados. Medicina Clínica (Barcelona) 2001; 116: 150-154

3. Mattick RP, Breen C, Kimber J, Davoli M. Methadone maintenance therapy versus no opioid replacement therapy for opioid dependence (Cochrane review). Cochrane library. Issue 4, 2002.

4. Ball JC, Ross A. The effectiveness of methadone maintenance treatment. Nueva York: Springer-Verlag, 1991

5. D'Aunno T, Vaughm C. Variations in methadone maintenance treatment practices. JAMA 1992; 267: 253-258.

6. Marsch LA. The efficacy of methadone maintenance interventions in reducing opiate use, HIV risk behavior and criminality: a meta-analysis. Addiction 1998; 93: 515-532.

7. Fernández Miranda JJ, González G-Portilla MP, Saiz Martínez PA, Gutiérrez Cienfuegos E, Bobes García J. Calidad de vida y severidad de la adicción en heroinómanos en mantenimiento prolongado con metadona. Adicciones 1999; 11(1): 43-52

8. Magura S, Nwakeze PC, Demsky S. Pre- and in-treatment predictors of retention in methadone treatment using survival analysis. Addiction 1998; 93: 51-60

9. Kosten TR, Rounsaville BJ, Kleber HD. Multidimensionality and prediction of treatment outcome in opioid addicts: 2,5-year follow-up. Comprehensive Psychiatry 1987; 28: 3-13.

10. McLellan AT, Arndt IO, Metzger DS, Woody GE, O'Brien CP. The effects of psychosocial services in substance abuse treatment. JAMA 1993; 269: 1953-1959.

11. Maddux JF, Desmond DP. Outcomes of methadone maintenance 1 year after admission. J Drug Issues 1997; 27: 225-238.

12. Yancovitz SR, Des Jarlais DC. Abdul-Quader AS, Peyser NP, Drew E, Friedmann P, Trigg HL, Robinson JV. A randomized trial of an interim methadone maintenance clinic. Am J Public Health 1991; 81: 1185-1194.

13. Dunteman GH, Condelli WS, Fairbank JA. Predicting cocaine use among methadone patients: analysis of 
findings from a national study. Hospital Community Psychiatry 1992; 43: 608-611.

14. Tschan F, Tueller N. Health status of methadone recipients before entering a methadone program. Schweiz Med Wochenschr 1992; 122: 1758-1769.

15. Fernández Miranda JJ, González G-Portilla MP, Saiz Martínez PA, Gutiérrez Cienfuegos E, Bobes García J. Consumo de drogas ilegales y alcohol antes y a lo largo de 6 años de mantenimiento con metadona y variables relacionadas. Adicciones 2001; 13(2): 179-188.

16. Grella CE, Wugalter SE, Anglin MD. Predictors of treatment retention in enhanced and standard methadone maintenance treatment for HIV risk reduction. J Drug Issues 1997; 27: 203-224.

17. Magura S, Siddiqui Q, Freeman RC, Lipton DS. Changes in cocaine use after entry in methadone treatment. Int J Addictions 1991; 10: 31-45.

18. Alterman Al, Rutherford MJ, Cacciola JS, McKay JR, McLellan T. Response to methadone maintenance and counseling in antisocial patients with and without major depression. J Nervous and Mental Disease 1996; 184: 695-702.

19. Fernández Miranda JJ, Marina González PA. Manual de los tratamientos de mantenimiento con opiáceos. Oviedo: Ed Gofer, 1999.

20. Fernández Miranda JJ, González G-Portilla MP, Saiz Martínez PA, Gutiérrez Cienfuegos E, Bobes García J. Influencia de los trastornos psiquiátricos en la efectividad de un programa de mantenimiento prolongado con metadona. Actas Españolas de Psiquiatría 2001; 29 (4): 228-232.
21. Friedman E, Glickman T. Prediction to succesful treatment outcome by client characteristics and retention in treatment in adolescent drug treatment programs: A large scale cross validation study. J Drug Education 1986; 16: 149-165.

22. Maddux JF, Prihoda TJ, Desmond DP. Methadone dose and urine morphine. J Substance Abuse Treat 1991; 8: 195-201.

23. Strain EC, Stitzer ML, Liebson IA, Bigelow GE. Doseresponse effects of methadone in the treatment of opioid dependence. Ann Intern Med 1993; 119: 23-27.

24. Batten HL. Drug services research survey. Phase I final report: Non correctional facilities . Rockville: N.I.D.A., 1991.

25. Caplehorn JRM, Mc Neil DR, Kleinbaum DG. Clinic policy and retention in methadone maintenance. Int $\mathrm{J}$ Addictions 1993; 28: 73-90.

26. Parrino MW. Sstate methadone treatment guidelines. U.S. department of health and human services. Rockville: Substance Abuse and Mental Health Services Administration Center, 1992.

27. Fernández Miranda JJ, Gutiérrez Cienfuegos E, Marina González., P. Actuaciones clínicas en trastornos adictivos. Madrid: Ed Aula Médica, 2002

28. Mira JJ, Lorenzo S, Rguez-Marín E, et al. Concepto y modelos de calidad. Hacia una definición de calidad asistencial. Papeles Psicol 1999; 74: 3-11.

29. Donabedian A. La calidad de la atención médica. Definición y métodos de evaluación. Méjico: Prensa mexicana, 1984 